\title{
Physically Realizable Parallel Decompositions of Experimentally Determined Mueller Matrices
}

\author{
Ignacio San José ${ }^{1}$ and José J. Gil ${ }^{2 *}$ \\ ${ }^{1}$ Instituto Aragonés de Estadística, Gobierno de Aragón, Bernardino Ramazzini 5, 50015 Zaragoza, Spain \\ ${ }^{2}$ Department of Applied Physics, University of Zaragoza, Pedro Cerbuna 12, 50009 Zaragoza, Spain \\ *ppgil@unizar.es
}

\begin{abstract}
Parallel decompositions have proved to be powerful tools for the analysis and interpretation of the physical information provided by Mueller matrices experimentally obtained. In this work, the procedure for the so-called arbitrary decomposition of Mueller matrices is validated through its application to a set of representative experimentally determined Mueller matrices. The covariance and passivity criteria required for the physical realizability of the Mueller matrices involved in the parallel decompositions are integrated in the procedure, which ensures the physical consistency of the results presented.
\end{abstract}

KEYWORDS: Mueller matrix; polarization

\section{Introduction}

A Mueller matrix $\mathbf{M}$ is a $4 \times 4$ matrix that transforms the Stokes vector $\mathbf{s}$ associated with an input electromagnetic wave into the Stokes vector $\mathbf{s}^{\prime}=\mathbf{M} \mathbf{s}$ of the output wave. When $\mathbf{M}$ preserves the degree of polarization of totally polarized input states (that is, $\mathbf{s}^{\prime}$ is totally polarized for any totally polarized $\mathbf{s}$ ) $\mathbf{M}$ is called pure (also nondepolarizing or Mueller-Jones matrix). Hereafter, when it is appropriate to indicated that $\mathbf{M}$ is pure, we will denote it as $\mathbf{M}_{J}$

In general, Mueller matrices can produce depolarization, in which case they can be submitted to parallel decompositions in order to analyze, classify and interpret them [1-6]. In particular, the procedure for the arbitrary decomposition of $\mathbf{M}$ [7] allows for infinite possible parallel decompositions of $\mathbf{M}$ in terms of convex sums of pure Mueller matrices.

Any Mueller matrix can be expressed in the following convenient manner [8],

$$
\begin{gathered}
\mathbf{M}=m_{00} \hat{\mathbf{M}}, \quad \hat{\mathbf{M}} \equiv\left(\begin{array}{cc}
1 & \mathbf{D}^{T} \\
\mathbf{P} & \mathbf{m}
\end{array}\right), \\
\mathbf{m} \equiv \frac{1}{m_{00}}\left(\begin{array}{lll}
m_{11} & m_{12} & m_{13} \\
m_{21} & m_{22} & m_{23} \\
m_{31} & m_{32} & m_{33}
\end{array}\right), \\
\mathbf{D} \equiv \frac{\left(m_{01}, m_{02}, m_{03}\right)^{T}}{m_{00}}, \mathbf{P} \equiv \frac{\left(m_{10}, m_{20}, m_{30}\right)^{T}}{m_{00}},
\end{gathered}
$$

where the superscript $T$ indicates transpose, $m_{00}$ is the mean intensity coefficient (MIC) (i.e. the transmittance or gain [9-14] of $\mathbf{M}$ for input unpolarized light), and $\mathbf{D}$ and $\mathbf{P}$ are the respective diattenuation and polarizance vectors of $\mathbf{M}$. The absolute values $D \equiv|\mathbf{D}|$ and $P \equiv|\mathbf{P}|$ are the diattenuation and the polarizance respectively.

The fact that a Mueller matrix is a phenomenological result of the ensemble average of a myriad of basic atomic or molecular lightmatter interactions leads to the so-called ensemble criterion [15$18]$, whose mathematical characterization is formulated by means of the nonnegativity of the four eigenvalues of the coherency matrix $\mathbf{C}$ associated with a given $\mathbf{M}[19,20]$. The explicit expression of the positive semidefinite Hermitian matrix $\mathbf{C}$ in terms of the elements of $\mathbf{M}$ can be found, for instance in Refs. [18,21]. Leaving aside the fact that the eigenvalues of an experimentally obtained C can be calculated numerically, equivalent sets of four explicit algebraic inequalities were presented in [12] and [22].

The fact that each molecular basic interaction is essentially passive (i.e., it does not amplify the intensity of light) entails that the Mueller matrix $\mathbf{M}$ representative of the macroscopic behavior satisfies a pair of necessary and sufficient inequalities called the passivity conditions [12]

$$
m_{00}(1+D) \leq 1, \quad m_{00}(1+P) \leq 1,
$$

which are necessary and sufficient for $\mathbf{M}$ to be susceptible to be decomposed, at least in one way, as a convex sum of pure and passive Mueller matrices. Note that pure Mueller matrices satisfy $P=D[23]$, so that the above pair of conditions becomes a single one.

In summary, the covariance and passivity inequalities required by the physical realizability of a Mueller matrix, constitute a set of six independent conditions for a $4 \times 4$ matrix to be a Mueller matrix.

The most general formulation of the arbitrary decomposition of a given $\mathbf{M}$ is described in [7], which is closely related to the procedure for polarimetric subtraction [24]. In the next section, the applicability of the arbitrary decomposition to experimental results obtained through Mueller polarimetry is verified by means of a set of representative examples.

\section{Physically realizable parallel decompositions of measured Mueller matrices}

To illustrate the flexible and powerful features of the arbitrary decomposition of Mueller matrices, a set of three experimentally measured Mueller matrices is considered.

In practice, it is the specific knowledge that the experimentalist has about the sample what focuses the particular choice of the nature of one or more components, thus determining certain 
constraints relative to the characteristics of the remaining components. Moreover, let us recall that a given depolarizing Mueller matrix can be obtained, in infinite ways, as the convex sum of different numbers of pure Mueller matrices, but the minimum number of components $r$ equals the rank of the associated coherency matrix, $r=\operatorname{rank} \mathbf{C}$ [25]. For the sake of concreteness and simplicity, in what follows the performed arbitrary decompositions will be realized with a number of $r$ pure components in the following manner

$$
\tilde{\mathbf{M}}=\sum_{i=1}^{r} k_{i} \tilde{\mathbf{M}}_{J i}, \sum_{i=1}^{r} k_{i}=1 .
$$

where $\tilde{\mathbf{M}}(\mathbf{M})$ is the passive representative of the Mueller matrix $\mathbf{M}$ under consideration. In accordance with the passivity conditions (2), $\tilde{\mathbf{M}}(\mathbf{M})$ is defined as the Mueller matrix proportional to $\mathbf{M}$ having the maximal mean intensity coefficient $\tilde{m}_{00}$ compatible with passivity, and is given by

$$
\tilde{\mathbf{M}} \equiv \frac{1}{1+X}\left(\begin{array}{cc}
1 & \mathbf{D}^{T} \\
\mathbf{P} & \mathbf{m}
\end{array}\right), \quad X \equiv \max (D, P) .
$$

The maximum number of constitutive retarders compatible with $\tilde{\mathbf{M}}$ [6] will be taken in each example, so that the decompositions will contain two retarders, namely $\tilde{\mathbf{M}}_{J 1}$ and $\tilde{\mathbf{M}}_{I 2}$ (both lacking of diattenuation and polarizance), and two diattenuators, namely $\tilde{\mathbf{M}}_{J 3}$ and $\tilde{\mathbf{M}}_{J 4}$, while the mean intensity coefficients of the said passive components take specific corresponding values.

For each example, certain meaningful parameters that are invariant with respect to dual-retarder transformations [26], will be calculated because they are useful for the interpretation of the polarimetric nature of the sample and to determine how close to a singular matrix $\mathbf{M}$ is [27]. Such quantities are the diattenuation $D$, the polarizance $P$ and det $\hat{\mathbf{M}}$. For more detailed analyses, other additional invariant parameters may be considered like, for instance, the degree of spherical purity $P_{S}$ [28], and the depolarization index $P_{\Delta}$ [29] (also called degree of polarimetric purity [25]) .

\subsection{Example 1}

The following experimentally determined Mueller matrix $\mathbf{M}_{1}$ corresponds to the reflection (angle of incidence $50^{\circ}$ ) of a light probe of $632.8 \mathrm{~nm}$ (He-Ne laser) on a steel specimen with surface roughness of $0.256-\mu \mathrm{m}$, and with a $\mathrm{MgF}_{2}$ film thickness of $89 \mathrm{~nm}$ [30],

$$
\mathbf{M}_{1}=\left(\begin{array}{cccc}
1.0000 & 0.1631 & -0.0322 & 0.0802 \\
0.0083 & 0.4038 & 0.2555 & -0.2158 \\
-0.0026 & 0.4297 & -0.1376 & 0.2016 \\
-0.0116 & 0.0597 & -0.3175 & -0.3690
\end{array}\right) .
$$

The eigenvalues of the coherency matrix $\mathbf{C}$ associated with $\mathbf{M}_{1}$ have the nonnegative values

$$
\hat{\lambda}_{1}=0.6270, \hat{\lambda}_{2}=0.1888, \hat{\lambda}_{3}=0.1292, \hat{\lambda}_{4}=0.0550,
$$

showing that $\mathbf{M}_{1}$ satisfies the covariance conditions and that $r=\operatorname{rank} \mathbf{C}=4$ (within the assumed experimental accuracy limits), that is, any arbitrary decomposition should include four parallel components. The calculated values for the additional invariants of $\mathbf{M}_{1}$ are

$$
D=0.1846, P=0.0145, \operatorname{det} \hat{\mathbf{M}}_{1}=0.1176 .
$$

Note that in this case $D>P$, so that the passive representative of $\mathbf{M}_{1}$ is given by

$$
\tilde{\mathbf{M}}_{1}=\frac{1}{1+D} \hat{\mathbf{M}}_{1}=\left(\begin{array}{cccc}
0.8442 & 0.1377 & -0.0272 & 0.0677 \\
0.0070 & 0.3409 & 0.2157 & -0.1822 \\
-0.0022 & 0.3627 & -0.1162 & 0.1702 \\
-0.0098 & 0.0504 & -0.2680 & -0.3115
\end{array}\right)
$$

Through the procedure for the arbitrary decomposition [7], a physically realizable parallel decomposition of $\mathbf{M}_{1}$ of the form (3) is the following

$$
\begin{aligned}
\tilde{\mathbf{M}}_{J 1} & =\left(\begin{array}{rrrr}
1.0000 & 0.0000 & 0.0000 & 0.0000 \\
0.0000 & 0.3203 & 0.6300 & -0.7074 \\
0.0000 & 0.8373 & 0.1610 & 0.5225 \\
0.0000 & 0.4431 & -0.7597 & -0.4760
\end{array}\right), \\
\tilde{\mathbf{M}}_{J 2} & =\left(\begin{array}{rrrr}
1.0000 & 0.0000 & 0.0000 & 0.0000 \\
0.0000 & 0.9744 & -0.1341 & -0.1803 \\
0.0000 & -0.0825 & -0.9598 & 0.2682 \\
0.0000 & -0.2091 & -0.2465 & -0.9463
\end{array}\right), \\
\tilde{\mathbf{M}}_{J 3} & =\left(\begin{array}{rrrr}
0.6532 & 0.3064 & -0.0605 & 0.1507 \\
0.2774 & 0.3255 & -0.0377 & 0.5254 \\
0.1360 & 0.4399 & 0.3158 & -0.1781 \\
-0.1576 & -0.3176 & 0.4571 & 0.1464
\end{array}\right), \\
\tilde{\mathbf{M}}_{J 4} & =\left(\begin{array}{rrrr}
0.5527 & 0.3953 & -0.0780 & 0.1944 \\
-0.3577 & -0.4652 & 0.1285 & -0.0195 \\
-0.2096 & -0.2058 & -0.2006 & -0.2583 \\
0.1679 & 0.0529 & -0.2338 & 0.2758
\end{array}\right),
\end{aligned}
$$

with respective coefficients

$$
k_{1}=0.3713, k_{2}=0.2270, k_{3}=0.2373, k_{4}=0.1644 \text {. }
$$

\subsection{Example 2}

Let us now analyze the case of the experimentally determined Mueller matrix $\mathbf{M}_{2}$ of a sample of polyacrylamide gel measured in transmission with visible light [31]

$$
\mathbf{M}_{2}=\left(\begin{array}{rrrr}
1.0000 & -0.0312 & 0.0029 & -0.0066 \\
-0.0214 & 0.7678 & -0.0370 & 0.0204 \\
-0.0055 & 0.0230 & 0.1043 & -0.7735 \\
0.0014 & 0.0390 & 0.7972 & 0.1920
\end{array}\right),
$$

with corresponding invariants

$$
\begin{gathered}
\hat{\lambda}_{1}=0.8424, \hat{\lambda}_{2}=0.0806, \hat{\lambda}_{3}=0.0424, \hat{\lambda}_{4}=0.0346, \\
D=0.0320, P=0.0221, \operatorname{det} \hat{\mathbf{M}}_{2}=0.4900 .
\end{gathered}
$$

The passive representative of $\mathbf{M}_{2}$ is

$$
\tilde{\mathbf{M}}_{2}=\frac{1}{1+D} \hat{\mathbf{M}}_{2}=\left(\begin{array}{rrrr}
0.9690 & -0.0302 & 0.0028 & -0.0064 \\
-0.0207 & 0.7440 & -0.0359 & 0.0198 \\
-0.0053 & 0.0223 & 0.1011 & -0.7495 \\
0.0014 & 0.0378 & 0.7725 & 0.0860
\end{array}\right) \text {, }
$$

which admits, for instance, the following physically realizable parallel decomposition of the form (3) 


$$
\begin{aligned}
\tilde{\mathbf{M}}_{J 1} & =\left(\begin{array}{rrrr}
1.0000 & 0.0000 & 0.0000 & 0.0000 \\
0.0000 & 0.5691 & 0.1687 & -0.8048 \\
0.0000 & -0.7012 & -0.4116 & -0.5821 \\
0.0000 & -0.4295 & -0.8956 & -0.1159
\end{array}\right), \\
\tilde{\mathbf{M}}_{J 2} & =\left(\begin{array}{rrrr}
1.0000 & 0.0000 & 0.0000 & 0.0000 \\
0.0000 & 0.9649 & 0.1710 & -0.1991 \\
0.0000 & -0.2588 & 0.7459 & -0.6137 \\
0.0000 & 0.0436 & 0.6437 & 0.7640
\end{array}\right), \\
\tilde{\mathbf{M}}_{J 3} & =\left(\begin{array}{rrrr}
0.8852 & -0.1119 & 0.0104 & -0.0237 \\
0.2578 & -0.1915 & 0.8047 & 0.2944 \\
-0.0061 & 0.2433 & 0.3401 & -0.7717 \\
0.1117 & -0.8288 & -0.0847 & -0.2877
\end{array}\right), \\
\tilde{\mathbf{M}}_{J 4} & =\left(\begin{array}{rrrr}
0.9538 & -0.0451 & 0.0042 & -0.0095 \\
-0.0438 & 0.8173 & -0.3121 & 0.3796 \\
-0.0096 & 0.3831 & -0.0569 & -0.8704 \\
-0.0114 & 0.3080 & 0.8983 & 0.0767
\end{array}\right),
\end{aligned}
$$

with respective coefficients

$$
k_{1}=0.1798, k_{2}=0.2444, k_{3}=0.0642, k_{4}=0.5116 .
$$

\subsection{Example 3}

Here we consider the case of the Mueller matrix $\mathbf{M}_{3}$ obtained by processing data obtained through imaging polarimetry, which were submitted to subsequent data processing techniques, and that corresponds to the nervure of a leaf [32]

$$
\mathbf{M}_{3}=\left(\begin{array}{rrrr}
1.0000 & 0.0227 & -0.0031 & -0.0028 \\
0.0077 & 0.2066 & -0.0038 & -0.0096 \\
0.0009 & -0.0121 & -0.2225 & -0.0024 \\
0.0035 & 0.0188 & -0.0082 & -0.1306
\end{array}\right),
$$

whose considered invariants take the values

$$
\begin{gathered}
\hat{\lambda}_{1}=0.3904, \hat{\lambda}_{2}=0.2222, \hat{\lambda}_{3}=0.2133, \hat{\lambda}_{4}=0.1741, \\
D=0.0231, P=0.0085 \text {, det } \hat{\mathbf{M}}_{3}=0.0056 \text {. }
\end{gathered}
$$

The passive representative of $\mathbf{M}_{3}$ is

$$
\tilde{\mathbf{M}}_{3}=\frac{1}{1+D} \hat{\mathbf{M}}_{3}=\left(\begin{array}{rrrr}
0.9774 & 0.0222 & -0.0030 & -0.0027 \\
0.0075 & 0.2019 & -0.0037 & -0.0094 \\
0.0009 & -0.0119 & -0.2175 & -0.0023 \\
0.0034 & 0.0184 & -0.0080 & -0.1277
\end{array}\right)
$$

which admits, for instance, the following physically realizable parallel decomposition of the form (3)

$$
\begin{aligned}
\tilde{\mathbf{M}}_{J 1} & =\left(\begin{array}{rrrr}
1.0000 & 0.0000 & 0.0000 & 0.0000 \\
0.0000 & 0.5372 & -0.5298 & -0.6563 \\
0.0000 & 0.0786 & -0.7433 & 0.6643 \\
0.0000 & -0.8398 & -0.4085 & -0.3577
\end{array}\right), \\
\tilde{\mathbf{M}}_{J 2} & =\left(\begin{array}{rrrr}
1.0000 & 0.0000 & 0.0000 & 0.0000 \\
0.0000 & -0.1571 & 0.9185 & 0.3628 \\
0.0000 & 0.0148 & -0.3652 & 0.9308 \\
0.0000 & 0.9874 & 0.1516 & 0.0438
\end{array}\right),
\end{aligned}
$$

$$
\begin{aligned}
\tilde{\mathbf{M}}_{J 3} & =\left(\begin{array}{rrrr}
0.9627 & 0.0367 & -0.0050 & -0.0045 \\
-0.0313 & -0.7016 & 0.4559 & 0.4757 \\
-0.0083 & -0.2569 & 0.4506 & -0.8103 \\
-0.0184 & -0.6070 & -0.7174 & -0.2066
\end{array}\right), \\
\tilde{\mathbf{M}}_{J 4} & =\left(\begin{array}{rrrr}
0.9446 & 0.0546 & -0.0074 & -0.0067 \\
0.0487 & 0.7727 & -0.5419 & 0.0284 \\
0.0089 & 0.0387 & 0.0050 & -0.9422 \\
0.0250 & 0.5418 & 0.7717 & 0.0262
\end{array}\right),
\end{aligned}
$$

with respective coefficients

$$
k_{1}=0.2952, k_{2}=0.2350, k_{3}=0.1917, k_{4}=0.2780 \text {. }
$$

\section{References}

1. J. M. Correas, P. A. Melero, J. J. Gil, “Decomposition of Mueller matrices into pure optical media," Monog. Sem. Mat. G. Galdeano 27, 23-240 (2003).

2. J. J. Gil, I. San José, R. Ossikovski, "Serial-parallel decompositions of Mueller matrices," J. Opt. Soc. Am. A. 30, 32-50 (2013).

3. J. J. Gil, "Parallel decompositions of Mueller matrices and polarimetric subtraction," EPJ Web of Conferences 5, 04002-3 (2010).

4. R. Ossikovski, E. Garcia-Caurel, M. Foldyna, J. J. Gil, "Application of the arbitrary decomposition to finite spot size Mueller matrix measurements," Appl. Opt. 53, 6030-6036 (2014).

5. J. J. Gil, "Invariant quantities of a Mueller matrix under rotation and retarder transformations," J. Opt. Soc. Am. A 33, 52-58 (2016).

6. I. San José, J. J. Gil, "Retarding parallel components of a Mueller matrix," Opt. Commun. In press (2019).

7. J. J. Gil, I. San José, "Arbitrary decomposition of a Mueller matrix," 44, 5715-5718 (2019)

8. Z-F Xing, "On the deterministic and non-deterministic Mueller matrix," J. Mod Opt. 39, 461-484 (1992).

9. R. Barakat, "Conditions for the physical realizability of polarization matrices characterizing passive systems," J. Mod. Opt. 34, 15351544 (1987).

10. C. Brosseau, R. Barakat, "Jones and Mueller polarization matrices for random media," Opt. Commun. 84, 127-132 (1991).

11. A. B. Kostinski, R. C. Givens, "On the gain of a passive linear depolarizing system," J. Mod. Opt. 39, 1947-1952 (1992).

12. J. J. Gil, "Characteristic properties of Mueller matrices," J. Opt. Soc. Am. A 17, 328-334 (2000).

13. V. Devlaminck, P. Terrier, "Non-singular Mueller matrices characterizing passive systems," Optik 121, 1994-1997 (2010).

14. J. J. Gil, "Transmittance constraints in serial decompositions of Mueller matrices. The arrow form of a Mueller matrix," J. Opt. Soc. Am. A 30, 701-707 (2013).

15. J. J. Gil, E. Bernabéu, "A depolarization criterion in Mueller matrices," Opt. Acta 32, 259-261 (1985).

16. S.R. Cloude, "Group theory and polarization algebra", Optik 75, 2636 (1986).

17. K. Kim, L. Mandel, E. Wolf "Relationship between Jones and Mueller matrices for random media," J. Opt. Soc. Am. A 4, 433-437 (1987). 
18. J. J. Gil, R. Ossikovski, Polarized Light and the Mueller Matrix Approach. (CRC Press 2016).

19. S. R. Cloude, "Group theory and polarisation algebra," Optik 75, 2636 (1986).

20. P. M. Arnal, Modelo matricial para el estudio de fenómenos de polarización de la luz. PhD Thesis. University of Zaragoza. 1990.

21. S. R. Cloude, Polarisation: Applications in Remote Sensing, (Oxford University Press, 2009).

22. J. J. Gil, I. San José, "Explicit algebraic characterization of Mueller matrices," Opt. Lett. 39, 4041-4044 (2014).

23. J. J. Gil, Determination of polarization parameters in matricial representation. Theoretical contribution and development of an automatic measurement device. PhD thesis, University of Zaragoza, 1983. Available at http://zaguan.unizar.es/record/10680/files/TESIS2013-057.pdf

24. J. J. Gil and I. San José, "Polarimetric subtraction of Mueller matrices," J. Opt. Soc. Am. A 30, 1078-1088 (2013).
25. J. J. Gil, "Polarimetric characterization of light and media," Eur. Phys. J. Appl. Phys. 40, 1-47 (2007).

26. J. J. Gil, "Invariant quantities of a Mueller matrix under rotation and retarder transformations," J. Opt. Soc. Am. A 33, 52-58 (2016).

27. J. J. Gil, R. Ossikovski, I. San José, "Singular Mueller matrices," J. Opt. Soc. Am. A 33, 600-609 (2016)

28. J. J. Gil, "Components of purity of a Mueller matrix," J. Opt. Soc. Am. A 28, 1578-1585 (2011).

29. J. J. Gil, E. Bernabéu, "Depolarization and polarization indices of an optical system," Opt. Acta 33, 185-189 (1986).

30. M. W. Williams, "Depolarization and cross polarization in ellipsometry of rough surfaces," Appl. Opt. 25, 3616-3622 (1986).

31. O. Arteaga, A. Canillas, "Pseudopolar decomposition of the Jones and Mueller-Jones exponential polarization matrices," J. Opt. Soc. Am. A. 26, 783-793 (2009).

32. C. Collet, J. Zallat, Y. Takakura, "Clustering of Mueller matrix images for skeletonized structure detection," Opt. Express 12, 1271- 1280 (2004). 